\title{
Compression of encrypted three-dimensional objects using digital holography
}

\author{
Thomas J. Naughton, MEMBER SPIE \\ National University of Ireland, Maynooth \\ Department of Computer Science \\ Maynooth, County Kildare, Ireland \\ E-mail: tom.naughton@may.ie \\ Bahram Javidi, FELLOW SPIE \\ University of Connecticut \\ Electrical and Computer Engineering \\ Department \\ 371 Fairfield Road, Unit 1157 \\ Storrs, Connecticut 06269
}

\begin{abstract}
We present the results of applying data compression techniques to encrypted three-dimensional objects. The objects are captured using phase-shift digital holography and encrypted using a random phase mask in the Fresnel domain. Lossy quantization is combined with lossless coding techniques to quantify compression ratios. Lossless compression alone applied to the encrypted holographic data achieves compression ratios lower than 1.05. When combined with quantization and an integer encoding scheme, this rises to between 12 and 65 (depending on the hologram chosen and the method of measuring compression ratio), with good decryption and reconstruction quality. Our techniques are suitable for a range of secure three-dimensional object storage and transmission applications. () 2004 Society of Photo-Optical Instrumentation Engineers. [DOI: $10.1117 / 1.1783280]$
\end{abstract}

Subject terms: digital holography; image compression; optical encryption; threedimensional image processing.

Paper 431009 received Oct. 2, 2003; revised manuscript received Feb. 20, 2004; accepted for publication Mar. 5, 2004.

\section{Introduction}

Many techniques for the optical encryption of image data have been proposed and implemented in recent years. ${ }^{1-12}$ Most perform encryption with a random phase mask positioned in the input, Fresnel, or Fraunhofer domain, or a combination of domains. These invariably produce a complex-valued encrypted image. Digital holography ${ }^{13-20}$ can be used to measure complex-valued wavefronts, and it has been applied to the encryption of 2-D conventional (real-valued) images. ${ }^{7-9}$ Of these, the techniques based on phase-shift interferometry ${ }^{15,17,20}$ (PSI) make good use of detector resources in that they capture on-axis encrypted digital holograms. ${ }^{8,9}$ The PSI technique has also been extended to the encryption of 3-D objects. ${ }^{10}$

The advantage of digital techniques over holographic encryption methods that use more traditional photorefractive media ${ }^{4,5}$ is that the resulting encrypted hologram can be easily stored electronically or transmitted over conventional communication channels. This motivates the study of how conventional compression techniques could be applied to digital holograms. Hologram compression differs to image compression principally because our holograms store 3-D information in complex-valued pixels, and secondly because of the inherent speckle content, which gives the holograms a white-noise appearance. It is not straightforward to remove the holographic speckle, because it actually carries 3-D information. The noisy appearance of digital holograms causes lossless data compression techniques to perform poorly on such inputs. ${ }^{21}$

In this paper, we apply quantization directly to the complex-valued holographic pixels. Treatments of quantization in holograms can be found in the literature ${ }^{22,23}$ and compression of real-valued ${ }^{24}$ and complex-valued ${ }^{21,25}$ digital holograms has received some attention to date. This in- troduces a third reason why compression of digital holograms differs from compression of digital images; a local change in a digital hologram will, in theory, affect the whole reconstructed object. Furthermore, when gauging the errors introduced by lossy compression, we are not directly interested in the defects in the hologram itself, only in how compression noise affects the quality of reconstructions of the compressed 3-D object.

The structure of the paper is as follows. In Sec. 2, the hologram encryption procedure is outlined. In Sec. 3, we examine the amenability of encrypted digital holograms to lossless compression using four well-known techniques, and in Sec. 4 apply the lossy technique of quantization to the real and imaginary components of each encrypted holographic pixel. We combine quantization with lossless compression in Sec. 5 to achieve far better compression performance than using either technique alone, and we conclude in Sec. 6.

\section{Digital Hologram Encryption}

The encrypted complex-valued holograms are captured using an optical setup (shown in Fig. 1) based on a MachZehnder interferometer architecture. ${ }^{26,27}$ A linearly polarized argon ion $(514.5 \mathrm{~nm})$ laser beam is divided into object and reference beams, both of which are spatially filtered and expanded. The first beam illuminates the 3-D object placed at a distance $d_{1}+d_{2}$ from a 10-bit 2028 $\times 2044$-pixel Kodak Megaplus CCD camera. A random phase mask is placed a distance $d_{1}$ from the 3-D object. Due to free-space propagation, and under the Fresnel approximation, ${ }^{28-30}$ the signal at the detector plane $H_{\mathrm{E}}(x, y)$ is given by the superposition integral 


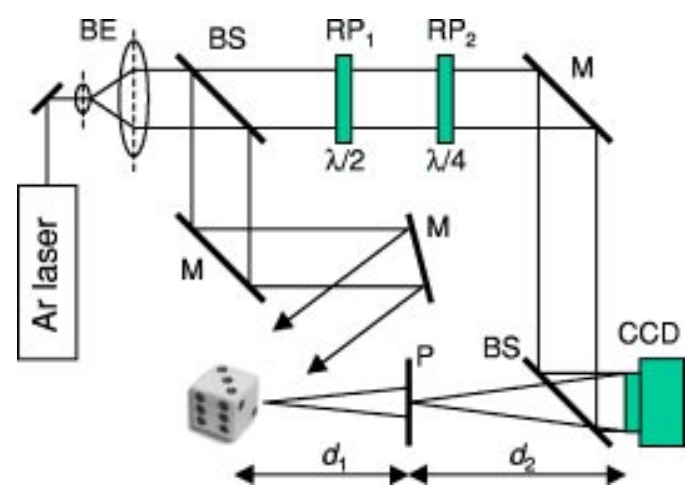

Fig. 1 Experimental setup for 3-D object encryption using phaseshift digital holography: BE, beam expander; BS, beamsplitter; $M$, mirror; RP, retardation plate; P, phase mask.

$$
\begin{aligned}
H_{\mathrm{E}}(x, y)= & \frac{-\mathrm{i}}{\lambda d_{2}} \exp \left(\mathrm{i} \frac{2 \pi}{\lambda} d_{2}\right) \iint_{-\infty}^{\infty} \exp \left[\mathrm{i} \Phi\left(x^{\prime}, y^{\prime}\right)\right] \\
& \times A_{\mathrm{M}}\left(x^{\prime}, y^{\prime}\right) \exp \left[\mathrm{i} \phi_{\mathrm{M}}\left(x^{\prime}, y^{\prime}\right)\right] \\
& \times \exp \left\{\mathrm{i} \frac{\pi}{\lambda d_{2}}\left[\left(x-x^{\prime}\right)^{2}+\left(y-y^{\prime}\right)^{2}\right]\right\} \mathrm{d} x^{\prime} \mathrm{d} y^{\prime},
\end{aligned}
$$

where $A_{\mathrm{M}}$ and $\phi_{\mathrm{M}}$ are the amplitude and phase, respectively, of the signal in the plane of, but immediately before, the random phase mask $\Phi$. The integral $H_{\mathrm{E}}(x, y)$ will have both its amplitude and phase modulated by the mask and will have a dynamic range suitable for capture by a CCD camera.

The reference beam passes through half-wave plate $\mathrm{RP}_{1}$ and quarter-wave plate $\mathrm{RP}_{2}$. This linearly polarized beam can be phase-modulated by rotating the two retardation plates. Through permutation of the fast and slow axes of the plates we can achieve phase shifts of $0,-\pi / 2,-\pi$, and $-3 \pi / 2$. The reference beam combines with the light diffracted from the object and forms an interference pattern in the plane of the camera. At each of the four phase shifts we record an interferogram. Using these four intensity images, the complex-valued camera-plane wavefront can be approximated in a computer to good accuracy using PSI.

Digital holograms of five reasonably diffuse 3-D objects were used in the experiments. ${ }^{21}$ Figure 2(a) shows one of the objects. This bolt had approximate dimensions of $5 \mathrm{~mm} \times 5 \mathrm{~mm} \times 5 \mathrm{~mm}$, and was positioned $d_{1}+d_{2}$ $=390 \mathrm{~mm}$ from the $2028 \times 2044$ pixel camera. The intensity image in Fig. 2(a) is reconstructed from a digital hologram captured using a version of the apparatus shown in Fig. 1 that did not contain a random phase mask. ${ }^{26,27}$ These reconstructions serve as ground truth data when quantifying lossy compression errors later in the paper.

In our experiments we use digital holograms that have been captured optically using the apparatus in Fig. 1 without the phase mask, ${ }^{26,27}$ and we encrypt them on a computer using simulated free-space propagation. ${ }^{31}$ The phase mask used in the simulations is shown in Fig. 2(b). It consists of values chosen with uniform probability from the range $[0,2 \pi)$ using a pseudorandom number generator. The

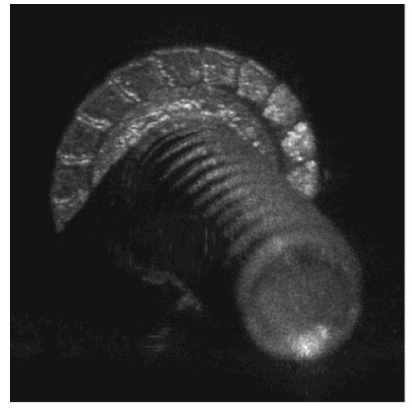

(a)

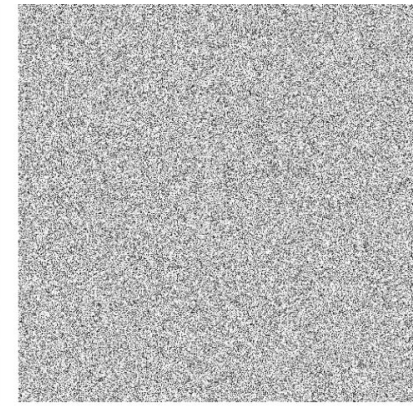

(b)
Fig. 2 The bolt object (a), and an example of a random-phase mask (b), used in the study.

mask has dimensions $2048 \times 2048$ pixels, and in the encryption experiments our digital holograms were enlarged from $2028 \times 2044$ pixels to these dimensions by padding with zeros. For our experiments, the mask was positioned as shown in Fig. 1 so that the ratio of the distances $d_{1}: d_{2}$ was 35:65. In Fig. 3 we show the amplitude and phase of the bolt hologram before encryption and after encryption.

\section{Lossless Compression of Encrypted Digital Holograms}

The digital holograms were treated as binary data streams, and compressed using the lossless data compression techniques of Lempeland Ziv (LZ77), Lempel-Ziv-Welch (LZW), Huffman, and Burrows-Wheeler (BW). The holograms are stored in native MATLAB floating-point representation with 8 bytes of real information and 8 bytes of imaginary information for each pixel. The holograms

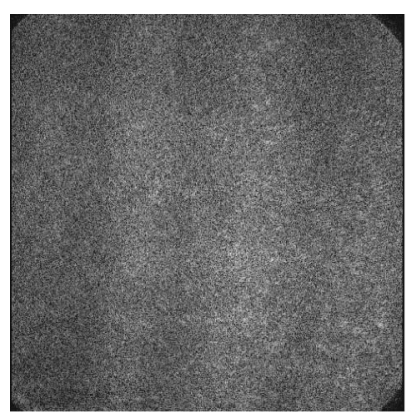

(a)

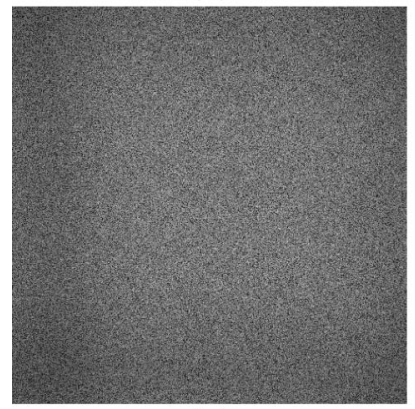

(c)

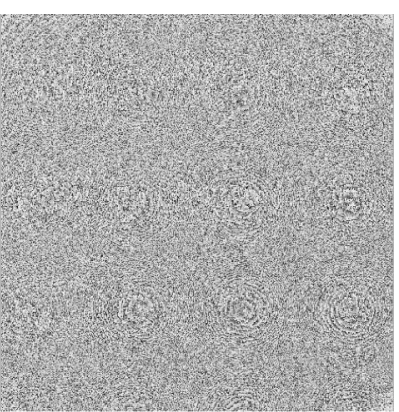

(b)

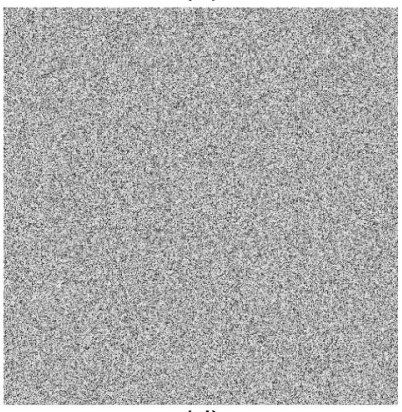

(d)
Fig. 3 The bolt hologram before and after encryption: (a) amplitude and (b) phase of the original hologram, and (c) amplitude and (d) phase of the encrypted hologram. 
Naughton and Javidi: Compression of encrypted three-dimensional objects . . .

Table 1 Lossless compression of encrypted digital holograms.

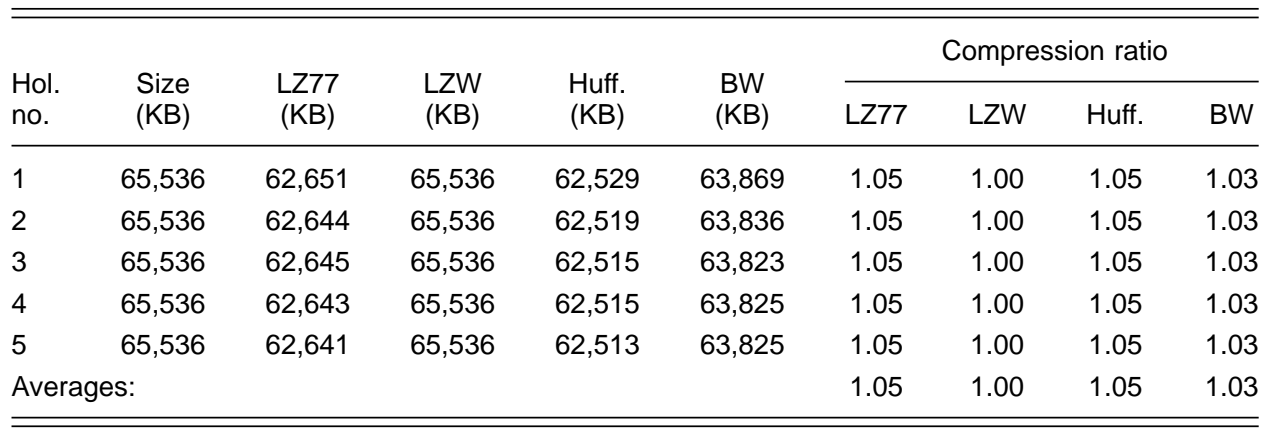

were first compressed without any encryption. Compressing separately the real and imaginary data streams achieves compression ratios in the range $[1.0,6.66],{ }^{21}$ where the compression ratio $r$ is calculated from

$r=\frac{\text { uncompressed size }}{\text { compressed size }}$.

Next, each of the five holograms was encrypted with the phase mask shown in Fig. 2(b). The encrypted holograms contained $2048 \times 2048$ pixels, and with 8 -byte real and imaginary values, this amounts to a file size of $65,536 \mathrm{~KB}$, where $1 \mathrm{~KB}=2^{10}$ bytes. The four lossless compression techniques were applied to each hologram (results shown in Table 1 -hologram 2 is the bolt).

From Table 1, very little redundancy or structure could be found in the encrypted hologram data. The random phase mask, combined with Fresnel propagation, is very effective at removing apparent structure from the hologram data. For some encrypted holograms, with LZW in particular, the compressed sizes were even larger than the uncompressed. In these cases the uncompressed encrypted file should be used, and a compression ratio of 1.0 is reported. These results illustrate the urgent need to explore lossy compression techniques suitable for encrypted digital holograms. One such lossy technique that has been successfully applied to 3-D digital holograms is quantization. ${ }^{21,25}$

\section{Quantization of Encrypted Digital Holograms}

The loss in reconstruction quality due to applying quantization to encrypted holograms was investigated. A combined rescale and quantization step was employed. The encrypted holograms were rescaled linearly to the square $[-1-\mathrm{i}, 1+\mathrm{i}]$ in the complex plane, and the real and imaginary components of each holographic pixel were then quantized.

We choose an odd number of quantization values (or levels) for each real and imaginary value: zero, and an equal number of positive and negative levels. As a result, $b$ bits encode $2^{b}-1$ levels. For example, two bits encode levels $\{-1,0,1\}$, three bits encode levels $\{-1,-2 / 3$, $-1 / 3,0,1 / 3,2 / 3,1\}$, and so on. The combined rescale-andquantization operation is defined for individual pixels as

$H^{\prime}(x, y)=\operatorname{round}\left[H(x, y) \times \sigma^{-1} \times \beta\right] \times \beta^{-1}$ and was applied to each pixel $(x, y)$ in the encrypted hologram $H$, where

$$
\begin{gathered}
\sigma=\max \{|\min [\operatorname{Im}(H)]|,|\max [\operatorname{Im}(H)]|, \\
|\min [\operatorname{Re}(H)]|,|\max [\operatorname{Re}(H)]|\},
\end{gathered}
$$

and where $\beta=2^{b-1}-1$. Here, $b$ represents the number of bits per real or imaginary value, $\max (\cdot)$ returns the maximum scalar in its $\operatorname{argument}(\mathrm{s})$, and $\operatorname{round}(\alpha)$ is defined as $\lfloor\alpha+0.5\rfloor$. After quantization, each real and imaginary value will be in the range $[-1,1]$.

The procedure for quantifying reconstruction loss due to quantization was as follows. An encrypted digital hologram $H(x, y)$ was quantized as $H^{\prime}(x, y)$ according to Eq. (3). The hologram was decrypted, and the entire hologram reconstructed, both processes being simulated using a computer. The quality of the reconstruction $U^{\prime}(x, y)$ was calculated by a comparison with the reconstruction $U_{0}(x, y)$ from an unencrypted (and unquantized) version of the digital hologram. The two reconstructions were compared in terms of the normalized rms (NRMS) difference of their intensities, defined as

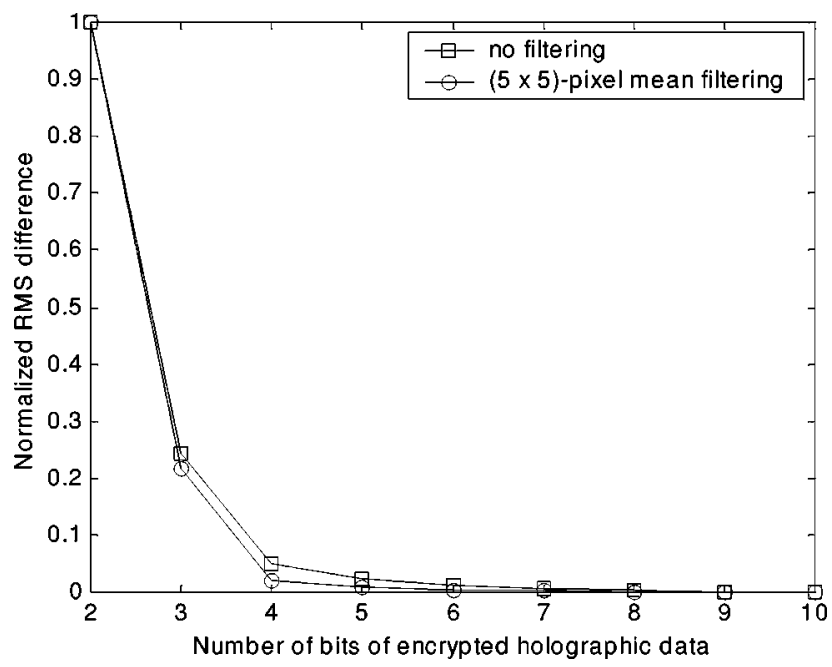

Fig. 4 NRMS intensity difference in decrypted and reconstructed 3-D bolt object plotted against quantization level. 


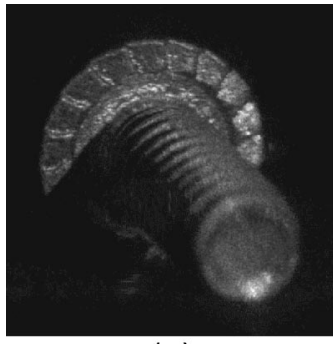

(a)

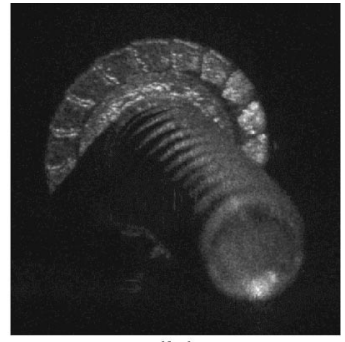

(b)

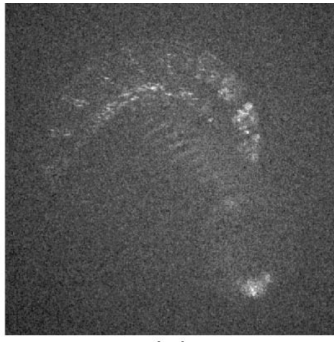

(c)

Fig. 5 Decrypted and reconstructed bolt object (with $5 \times 5$ pixel mean filtering) from an encrypted digital hologram with the following resolution in each real and imaginary value: (a) 4 bits, (b) 3 bits, (c) 2 bits.

$$
\begin{aligned}
D\left(U^{\prime}\right)= & \left(\sum_{m=0}^{N_{x}-1} \sum_{n=0}^{N_{y}-1}\left[\left|U_{0}(m, n)\right|^{2}-\left|U^{\prime}(m, n)\right|^{2}\right]^{2}\right. \\
& \left.\times\left\{\sum_{m=0}^{N_{x}-1} \sum_{n=0}^{N_{y}-1}\left[\left|U_{0}(m, n)\right|^{2}\right]^{2}\right\}^{-1}\right)^{1 / 2},
\end{aligned}
$$

where $(m, n)$ are discrete spatial coordinates in the reconstruction plane, and $N_{y}$ and $N_{x}$ are the height and width of the reconstructions, respectively. The slightest change to a digital hologram can result in an entirely different speckle pattern in the reconstruction domain. It could be argued that one should attempt to remove this somewhat quantizationinvariant speckle effect before measuring the quantization error. Therefore we also present the results of applying a mean filtering operation to both the original and compressed intensities prior to calculating the NRMS.

Figure 4 shows a plot of NRMS difference against number of bits per (real or imaginary) datum value in the encrypted hologram of the bolt object, with and without mean filtering over a neighborhood of $5 \times 5$ pixels. Figure 5 shows decrypted and reconstructed object intensities for the bolt for selected quantization resolutions. To some degree, the quantization noise is masked visually by the presence of speckle; this would not be the case if incoherent images were encrypted and quantized.

\section{Combining Quantization with Lossless Data Compression}

We perform two lossless compression steps on the quantized encrypted hologram data. In the first, we encode each quantized real and imaginary value with the minimum whole number of bytes required to represent it (if the value contains $b$ bits, then it requires $\lceil b / 8\rceil$ bytes). In the second, the real and imaginary streams were concatenated together and processed by one of the lossless techniques outlined earlier. Table 2 shows the results of this three-step compression process for the bolt hologram.

The PSI calculations contain trigonometric and division operations, which return values with theoretically infinite decimal (and binary) expansions that utilize completely the 8 -byte resolution of the encrypted digital hologram's real and imaginary values. (This is verified experimentally in Table 1, where little redundancy could be found in the 8-byte data.) However, it could be argued that four 10-bit intensity interferograms cannot be combined to create a digital hologram with more than 10 or 12 bits of meaningful information in each value. The standard portable encoding for a 10- or 12-bit value would be a 2-byte data type. Therefore, we also include in Table 2 (in parentheses) the calculations of the compression ratio where we assume that the original encrypted hologram could be effectively represented with only 2 bytes per real or imaginary value.

Compared to Table 1, Table 2 shows dramatic increases in compression ratio for all quantizations, and across all lossless compression algorithms. For example, with 3-bit quantization, a compression ratio of 65 (16, assuming 2-byte original values) is possible with LZW for reasonable decryption and reconstruction quality.

In order to quantify the gains made through lossless compression after quantization, we compare the lossless algorithms with the simple bit-packing technique. ${ }^{25}$ Bit pack-

\begin{tabular}{|c|c|c|c|c|c|c|c|c|c|}
\hline \multirow[b]{2}{*}{ Bits } & \multirow{2}{*}{$\begin{array}{l}\text { Size } \\
\text { (KB) }\end{array}$} & \multirow{2}{*}{$\begin{array}{c}\text { LZ77 } \\
\text { (KB) }\end{array}$} & \multirow{2}{*}{$\begin{array}{c}\text { LZW } \\
(\mathrm{KB})\end{array}$} & \multirow{2}{*}{$\begin{array}{l}\text { Huff. } \\
\text { (KB) }\end{array}$} & \multirow{2}{*}{$\begin{array}{l}\text { BW } \\
\text { (KB) }\end{array}$} & \multicolumn{4}{|c|}{ Compression ratio } \\
\hline & & & & & & LZ77 & LZW & Huff. & BW \\
\hline 2 & $65,536(16,384)$ & 47 & 42 & 1027 & 32 & $1394(349)$ & $1560(390)$ & $64(16)$ & $2048(512)$ \\
\hline 3 & $65,536(16,384)$ & 1138 & 1006 & 1317 & 1097 & $58(14)$ & $65(16)$ & $50(12)$ & $60(15)$ \\
\hline 4 & $65,536(16,384)$ & 2120 & 1963 & 1991 & 2084 & $31(7.7)$ & $33(8.3)$ & $33(8.2)$ & 31 (7.9) \\
\hline 5 & $65,536(16,384)$ & 3097 & 2969 & 3021 & 2985 & $21(5.3)$ & $22(5.5)$ & $22(5.4)$ & $22(5.5)$ \\
\hline 6 & $65,536(16,384)$ & 4003 & 4018 & 3923 & 3901 & $16(4.1)$ & $16(4.1)$ & $17(4.2)$ & $17(4.2)$ \\
\hline 7 & $65,536(16,384)$ & 4732 & 5124 & 4784 & 4795 & $14(3.5)$ & $13(3.2)$ & $14(3.4)$ & $14(3.4)$ \\
\hline 8 & $65,536(16,384)$ & 5460 & 6236 & 5613 & 5659 & $12(3.0)$ & $11(2.6)$ & $12(2.9)$ & $12(2.9)$ \\
\hline
\end{tabular}

Table 2 Lossless compression applied to encrypted and quantized hologram 2. 


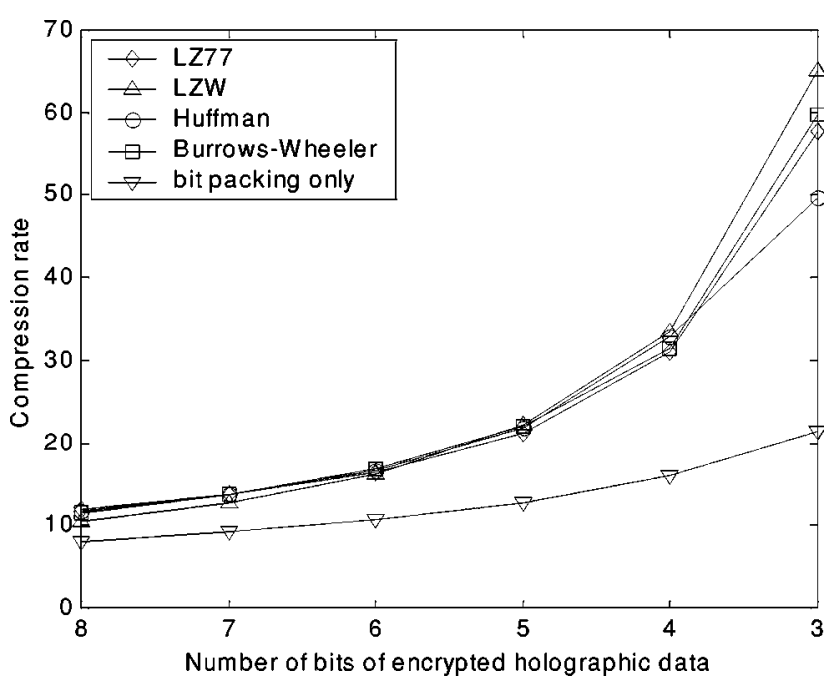

Fig. 6 Compression ratio for the bolt hologram, plotted against quantization combined with various lossless data compression techniques.

ing is the most basic step that could be applied to quantized data, and therefore serves to best quantify the compression ratio due to quantization alone. Bit packing uniformly reduces the length of each real and imaginary value's description, but does not exploit any redundancy or repetition in the data. In this technique, data reduction is performed by extracting the appropriate $b$ bits from each real and imaginary value. The data values are converted into integers in the range $\left[-2^{b-1}+1,2^{b-1}-1\right]$. The parity bit and loworder $b-1$ bits from each quantized value are accumulated in a bit buffer and packed into bytes. A digital hologram of $N \times N$ pixels at $b$-bit resolution requires exactly $\left\lceil 2 N^{2} b / 8\right\rceil$ packed bytes. This packed size is used to calculate the compression ratio.

The plot in Fig. 6 illustrates the improvement over bit packing that can be achieved by employing one of the more sophisticated lossless techniques to quantized holographic data. The lossless compression algorithms perform equally well on quantized data up to 4 bits. In such cases, one's choice of lossless algorithm would be determined by one's application requirements. For a secure 3-D digital hologram networking application, for example, one's concerns could include speed of the compressor and decompressor (in which case LZW/LZ77 might be preferable), reliability of the underlying network (in which case Huffman might be the only option), memory available to the compressor or decompressor, and whether the data are to be streamed or not (i.e., whether one wishes to start decompressing the data stream before the full stream has been received).

\section{Conclusion}

This paper has outlined the results of combining lossy and lossless techniques to the compression of encrypted digital holograms of 3-D objects. The optical encryption technique, based on phase-shift digital holography, is suitable for secure 3-D object storage and transmission applications. Lossless techniques, on their own, perform very poorly on encrypted (and unencrypted) digital hologram data because of their white noise characteristics. Quantization has been applied to good effect on the encrypted hologram data, and reductions to as few as 3 bits in each real and imaginary part have resulted in good decompressed and decrypted 3-D object reconstructions. Not only does quantization perform significant compression itself (measured through the use of a basic bit-packing algorithm), but it also reduces the number of symbols (for Huffman) and introduces structure into the bitstream (for LZ77 and LZW) to allow them to perform more effectively.

\section{Acknowledgments}

The authors wish to thank Enrique Tajahuerce and Yann Frauel for use of their hologram data. The first author acknowledges support from Enterprise Ireland.

\section{References}

1. B. Javidi and J. L. Horner, "Optical pattern recognition for validation and security verification," Opt. Eng. 33, 1752-1756 (1994).

2. P. Réfrégier and B. Javidi, "Optical image encryption based on input plane and Fourier plane random encoding," Opt. Lett. 20, 767-769 (1995).

3. L. G. Neto and Y. Sheng, "Optical implementation of image encryption using random phase encoding," Opt. Eng. 35, 2459-2463 (1996).

4. G. Unnikrishnan, J. Joseph, and K. Singh, "Optical encryption system that uses phase conjugation in a photorefractive crystal," Appl. Opt. 37, 8181-8186 (1998)

5. O. Matoba and B. Javidi, "Encrypted optical memory system using three-dimensional keys in the Fresnel domain," Opt. Lett. 24, 762764 (1999).

6. P. C. Mogensen and J. Glückstad, "Phase-only optical encryption," Opt. Lett. 25, 566-568 (2000).

7. B. Javidi and T. Nomura, "Securing information by use of digital holography," Opt. Lett. 25, 28-30 (2000).

8. S. Lai and M. A. Neifeld, "Digital wavefront reconstruction and its application to image encryption," Opt. Commun. 178, 283-289 (2000).

9. E. Tajahuerce, O. Matoba, S. C. Verrall, and B. Javidi, "Optoelectronic information encryption with phase-shifting interferometry," Appl. Opt. 39, 2313-2320 (2000).

10. E. Tajahuerce and B. Javidi, "Encrypting three-dimensional information with digital holography," Appl. Opt. 39, 6595-6601 (2000).

11. B. Hennelly and J. T. Sheridan, "Optical image encryption by random shifting in fractional Fourier domains," Opt. Lett. 28, 269-271 (2003).

12. N. K. Nishchal, J. Joseph, and K. Singh, "Fully phase encryption using fractional Fourier transform," Opt. Eng. 42, 1583-1588 (2003)

13. J. W. Goodman and R. W. Lawrence, "Digital image formation from electronically detected holograms," Appl. Phys. Lett. 11, 77-79 (1967).

14. M. A. Kronod, N. S. Merzlyakov, and L. P. Yaroslavskii, "Reconstruction of a hologram with a computer," Sov. Phys. Tech. Phys. 17, $333-$ 334 (1972).

15. J. H. Bruning, D. R. Herriott, J. E. Gallagher, D. P. Rosenfeld, A. D. White, and D. J. Brangaccio, "Digital wavefront measuring interferometer for testing optical surfaces and lenses," Appl. Opt. 13, 26932703 (1974)

16. T.-C. Poon and A. Korpel, "Optical transfer function of an acoustooptic heterodyning image processor," Opt. Lett. 4, 317-319 (1979).

17. J. Schwider, B. Burow, K. E. Elsner, J. Grzanna, and R. Spolaczyk, "Digital wavefront measuring interferometry: some systematic error sources," Appl. Opt. 22, 3421-3432 (1983).

18. L. Onural and P. D. Scott, "Digital decoding of in-line holograms," Opt. Eng. 26, 1124-1132 (1987).

19. U. Schnars and W. P. O. Jüptner, "Direct recording of holograms by a CCD target and numerical reconstruction," Appl. Opt. 33, 179-181 (1994).

20. I. Yamaguchi and T. Zhang, "Phase-shifting digital holography," Opt. Lett. 22, 1268-1270 (1997)

21. T. J. Naughton, Y. Frauel, B. Javidi, and E. Tajahuerce, "Compression of digital holograms for three-dimensional object reconstruction and recognition," Appl. Opt. 41, 4124-4132 (2002).

22. J. W. Goodman and A. M. Silvestri, "Some effects of Fourier domain phase quantization," IBM J. Res. Dev. 14, 478-484 (1970).

23. W. J. Dallas and A. W. Lohmann, "Phase quantization in holograms,' Appl. Opt. 11, 192-194 (1972).

24. T. Nomura, A. Okazaki, M. Kameda, Y. Morimoto, and B. Javidi, "Digital holographic data reconstruction with data compression," Proc. SPIE 4471, 235-242 (2001).

25. T. J. Naughton, J. B. M. Donald, and B. Javidi, "Efficient compres- 
sion of Fresnel fields for Internet transmission of three-dimensional images," Appl. Opt. 42, 4758-4764 (2003).

26. B. Javidi and E. Tajahuerce, "Three-dimensional object recognition by use of digital holography," Opt. Lett. 25, 610-612 (2000).

27. Y. Frauel, E. Tajahuerce, M.-A. Castro, and B. Javidi, "Distortiontolerant three-dimensional object recognition with digital holography," Appl. Opt. 40, 3887-3893 (2001).

28. J. W. Goodman, Introduction to Fourier Optics, 2nd ed., McGrawHill, New York (1996)

29. H. J. Caulfield, Ed., Handbook of Optical Holography, Academic Press, New York (1979).
30. A. D. McAulay, "Inverse computation of phase masks for two-layer diffractive optic element system," Proc. SPIE 4789, 99-105 (2002).

31. T. J. Naughton and B. Javidi, "Encryption and decryption of threedimensional objects using digital holography" (in preparation).

Thomas J. Naughton: Biography and photograph not available.

Bahram Javidi: Biography and photograph appear with the special section guest editorial in this issue. 